\title{
Semantic E-Learn Services and Intelligent Systems using Web Ontology
}

\author{
K.Vanitha, K.Yasudha \\ Assistant Professor, \\ Department of Computer Science, \\ GITAM University, Visakhapatnam.
}

\author{
Dr.M.Sri Venkatesh \\ Associate Professor, \\ Department of Computer Science, \\ GITAM University, Visakhapatnam
}

\author{
K.N.Sowjanya \\ Assistant Professor \\ Dept.IT, \\ GITAM University
}

\begin{abstract}
Present vision for the web is the semantic web in which information is given explicit meaning, making it easier for machines to automatically process and integrate information available on the web. It provides the information exactly. Now days, ontology is playing a major role in knowledge representation for the semantic web [1]. Ontology is a conceptualization of domain into a human understandable and machine readable or machine process able format consisting of entities, attributes, relationships and axioms. Ontology web language is designed for use by applications that need to process the content of information [22]. In this context many e-learning systems were proposed in the literature. Semantic Web technology may support more advanced Artificial intelligence problems for knowledge retrieval [20]. This paper aims at presenting an intelligent e-learning system from the literature.
\end{abstract}

Keywords-Semantic web; e-learning; Ontology Web Language (OWL); Ontology; OWL-S Service Ontology.

\section{INTRODUCTION}

The emergence of web technologies for data and knowledge interaction gives rise to the need for supportive frameworks for knowledge distribution. Semantic web in which information is given explicit meaning, making it easier for machines to automatically process and integrate information available on the web aimed at providing shared semantic spaces for web contents[12]. Now days with the rapid development of technology the learning methods have been changed. E-learning systems are taking prominent role in making the humans learning methods apart from the class room teaching irrespective of their age, income etc., in this scenario, in the literature there are many methods have been proposed and used [3]. Fayed et al proposed a model based on semantic web technology which is used by the Qatar university students and faculty of engineering [2]. Another intelligent web teacher system for learning personalization using semantic web model was proposed by Nicola, Gaeta1 [3] and there is an adaptive educational hypermedia systems [AEHS] by Metteo et al. This paper aims at presenting intelligent e-learning systems modeled by Fayed et.al and Nicola et al and Mateo et al.

\section{SEMANTIC WEB}

In recent years Semantic Web is the hottest topic in the area of AI and in the internet community. Semantic Web performs the meaning (semantics) of information and services on the web, and making it possible for the web to "understand" and satisfy the requests of people and machines to use the web content which is the idea of world wide web inventor Tim Berners-Lee. Semantic web builds an appropriate infrastructure for intelligent agents to verify the web, while performing complex actions for their users. Ultimately, Semantic Web is about how to implement reliable, large-scale interoperation of Web services, to make such services computer interpretable - to create a Web of machineunderstandable and interoperable services that intelligent agents can discover, execute and compose automatically [2].

The latest view of the semantic web has been changed as services. These services can be divided on two families "world services" and "web Services".

The example for a world service includes a shop, a museum, a restaurant, whose address type and description is accessible over the web. In contrast, a web service is a resource that can be automatically retrieved and invoked over the web [11]. Web service based applications can consider as conglomerates of independent, autonomous services developed by independent parties. Such components are not integrated at design time; they are integrated dynamically at runtime according to the current needs [15]. For example, an e-learning course can be assembled dynamically by composing learning objects stored in independent repositories.

\section{A. Meta data}

The preliminary source for performing semantic web operations is based on metadata. Metadata is "data about data". The aim of incorporating the Meta data is to find the data sources from the web, when end-user tries to search for information on the web [11]. Generally the data sources will be heterogeneous which belongs to different types i.e., unstructured, semi-structured and structured. Generally for the semantic web the data source will be a document, a web page, textual content, data, audio or video [8].

In the Semantic web, documents are marked up with semantic metadata which is machine-understandable about the human readable content of documents. The following are the different types for Meta data.

- Syntactic Metadata: The simplest form of metadata which describes non-contextual information about content and provides general information. 
- Structural Metadata: Provides the information regarding the structure of the content and describes how items are arranged.

- Semantic Metadata: This adds relationships, rules, and constraints to syntactic and structural metadata and describes contextually relevant or domain-specific information about content based on ontology [21].

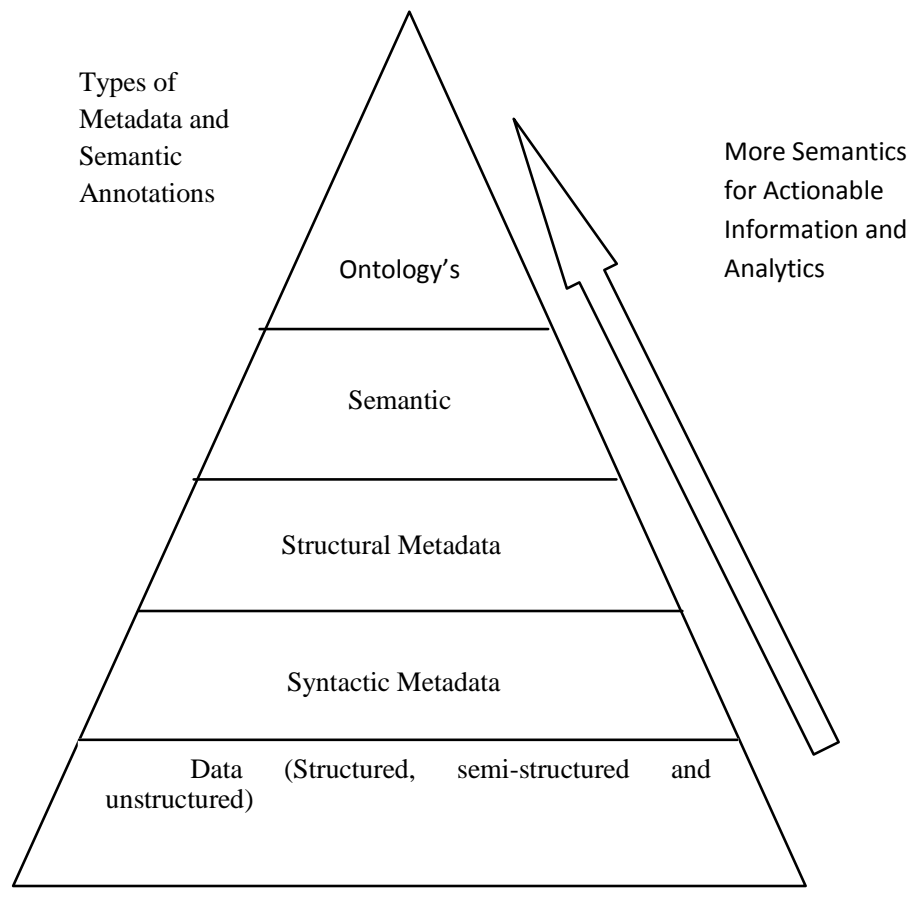

A. OWL-S Service Ontology

Fig1: Types of Metadata

OWL-D is an OWL service upper ontology that offers a Vocabulary that can be used in conjunction with OWL to describe services in an unambiguous, computer interpretable format. OWL-S was developed with the goal of allowing discovery, invocation, composition, and automatic monitoring of Web services (Martin et al, 2006). OWL-S treats service composition as processes. There is a very clear distinction among process properties, Structure, and implementation in OWL-S, which provides a way to model a process independently of its implementation.

The web service technology will revolutionize the way software is developed. Some of the potential benefits of the web services technologies are decentralization, speed, software packing and the other extreme web service technology has received a deal of criticism for providing an over simplified model. It leads out several fundamental concepts as Data definition, service invocation behavior mediation, composition and service guarantees.

The technology will allow a distributed and decentralized way of web services [11]. A positive effect of the increase of transactions through the web is forcing to adapt a more dynamic and user centered service model.

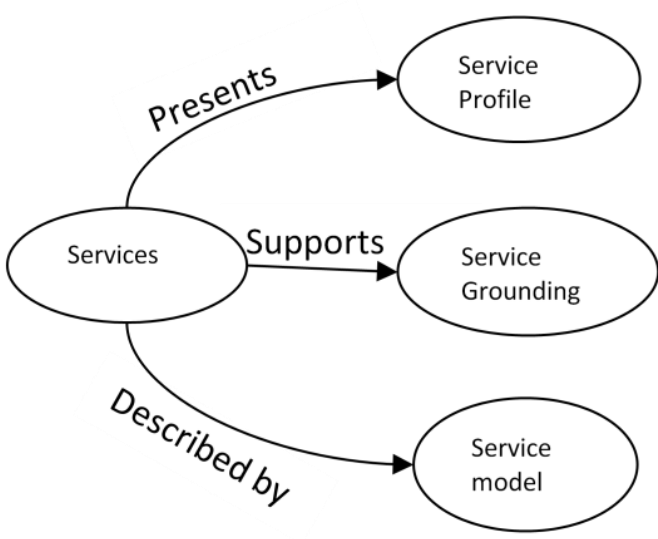

Fig:2 OWL-S Service Ontology

It is transforming response time into the competitive advantage. The web service compositional model has the potential to review the format and allow to be developed as service components. In over simplified model of concepts there are no domain specific data definitions. It is used to model the input and output of every application that is depending upon application domain.

\section{WEB ONTOLOGY}

Ontology is about the exact description of things and their relationships. Ontology's are considered one of the pillars of the Semantic Web; although they do not have a universally accepted definition According to Tom Gruber [17] ontology is a formal specification of a shared conceptualization [18]. For the web, ontology is about the exact description of web information and relationships between web information. The purpose of the Web Ontology domain is to be able to model the relationships between prominent web ontology's and map them onto equivalent freebase types and topics.

\section{AN ADAPTIVE EDUCATIONAL HYPERMEDIA SYSTEM (AEHS)}

The focus of Mateo et al. is on the aspects of personalization. They proposed a model as "An Adaptive Educational Hypermedia System" which supports the individual in the process of finding, selecting, accessing and retrieving web resources [2].

This model is based on the concepts of adaptive hypermedia system [19]. This adaptive hyper media system is in turn based on hypermedia system which was presented in brief in this paper.

\section{A. Personalization}

The goal of personalization in the Semantic web is to make easier the access to the right resources. This task entitles two processes [19] [5]. They are retrieval and presentation. Retrieval consists in finding or constructing the right resources when they are needed, either on demand otherwise, when the information arises in the work [8]. Personalization is a process of filtering the access to web content according to the individual needs and requirements of each particular user. 


\section{B. Adaptive Hypermedia System}

This enumerates the functionality of a hypermedia system which personalizes for the individual users.

\section{Hypermedia System}

A hypermedia system consists of documents which are connected by links[6]. Thus, there are mainly two aspects which can be adapted to the users: the content and the links.

\section{Content Level}

There are five methods identified for content level adaption.

- Additional explanation method which displays those parts of a document fits to user goals ,interest, tasks, knowledge etc.,

- Prerequisite explanations: in this method, the user model checks the prerequisites necessary to understand the content of the page.

- Comparative explanation : Comparative explanation is to explain new topics by stressing their relations to known topics

- Explanation variant: Explanation variants and extension to the prerequisite explanations

- Sorting: According to the need of the user, the different parts of the document are sorted.

Content level adaption methods will be implemented by the following techniques which deal with the knowledge. They are

- Conditional text: Information about a knowledge concept is divided in two different parts. Every part is defined with the knowledge.

- Stretch text: For some keywords of a document, according to the requirement of the user this technique provides longer descriptions.

- Page or page fragment variant: Different parts of the page are stored.

- Frame base fragments: this technique stores the page fragments into frames in a special order.

\section{E. Link level adaption}

Personalization for the user is being made through the link level adaption the following are the methods for navigating link level adaption[16].

i) Direct Guidance: "next best" and "page sequencing" are the two methods to guide the user sequentially through the hypermedia system [14]. "Nest best" provides nest button to navigate where page sequencing generates a reading sequence.

ii) Adaptive Sorting: "Similarity Sorting and "pre requisite sorting" are used based as the relevance system assumption by him/her, otherwise according to the prerequisite knowledge [8].

iii) Adaptive Hiding: Irrelevant information can be limited by making them unavailable or invisible. iv) Link Annotation: Several methods are available to annotate the educational area links for example traffic metaphor, where a red ball indicates lack of knowledge of understanding the pages yellow ball indicates that the link to pages are not recommended for reading[7] [9]. Green ball indicates links recommended pages.

v) Map Annotation: the same link annotation methods can be applied for maps.

\section{ADAPTIVE EDUCATIONAL}

\section{A. HYPERMEDIA SYSTEM METHODOLOGY (AEHSM) :}

A component based logical description of adaptive educational hypermedia system is proposed by Matteo et al [12]. This component based definition is based on the theory of diagnosis by Reiter [20].

\section{B. How it works?}

According to Matteo et al [12] AEHS was decomposed into basic components according to their roles. This uses a user model to model various characteristics of individual users or user groups. The adaptive functionality is provided by the organization of the document space and the user model [10].

This Adaptive Educational Hyper Media System is a quadruple. They are i) document Space (Docs), User Model (UM) Observations (OBS) and Adaption Component (AC) [22]. The document space and observations describe basic data and runtime data. This data will be processed by the other two. AEHS makes it Simple by annotating text using the traffic light metaphor. This can be extended by using Knowledge graph instead of domain graph [15]. This system is able to give a more differentiated traffic light annotation to hypertext links than simple [13]. It is able to recommend pages with green icon and to show which links lead to documents that will become understandable with dark orange icon and yellow icon is for the pages which might be understandable and red icon for which are not recommended yet. The representation of AEHS Simple and Knowledge graph with quadruple were presented in detail with examples.

a) Simple can annotate hypertext links by using the traffic light metaphor with two colors: red for nonrecommended, green for recommended pages.

i) DOCSs: This component is made of a set of $n$ constants and a finite set of predicates. Each of the constants represents a document in the document space (the documents are denoted by $D 1$, $D 2, \ldots, D n)$. The predicates define pre-requisite conditions, i.e. they state which documents need to be studied before a document can be learned, e.g. $\quad \operatorname{preq}(D i, D j)$ for certain $D i_{-}=D j$ means that $D j$ is a prerequisite for $D i$

ii) $U M s$ : it contains a set of $m$ constants, one for each individual user $U 1, U 2, \ldots, U m$.

iii) OBSs: A special constant (Visited) is used within the special predicate $o b s$ to denote whether a document has been visited: obs (Di, Uj, Visited $)$ is the observation that a document $D i$ has been visited by the user $U j$.

iv) ACs: This component contains constants and rules. One constant is used for describing the values of the "learning state" of the adaptive functionality, two constants (Green Icon 
and Red Icon) for representing values of the adaptive functionality. The learning state of a document is described by a set of rules of kind:

$$
\forall U_{i} \forall D_{j}\left(\forall D_{k} \operatorname{preq}\left(D_{j}, D_{k}\right) \Rightarrow \operatorname{obs}\left(D_{k}, U_{i}, \text { Visited }\right)\right) \Rightarrow
$$

learning-state $\left(D_{j}, U_{i}\right.$, Recommended for reading)

This component contains also a set of rules for describing the adaptive link annotation with traffic lights. Such rules are of kind:

$$
\begin{aligned}
& \forall U_{i} \forall D_{j} \text { learning-state }\left(D_{j}, U_{j} \text { Recommended for- reading }\right) \\
& \text { Ui,Green_icon) } \\
& \text { or of kind: }
\end{aligned}
$$
reading)

$\forall U_{i} \forall D_{j} \neg$ learning- $\operatorname{state}\left(D_{j}, \quad U_{i}\right.$, Recommended foricon)

$$
\Rightarrow \text { document annotation }\left(D_{j}, U_{i},\right. \text { Green }
$$

b) This simple AEHS can be extended by using a knowledge graph instead of a domain graph. The system, called Simple1, is able to give a more differentiated traffic light annotation to hypertext links than Simple [8]. It is able to recommend pages (green icon), to show which links lead to documents that will become understandable (dark orange icon), which might be understandable (yellow icon), or which are not recommended yet (red icon) [21]. Let us represent Simple1 by a quadruple (DOCSs $1, U M s 1, O B S s 1, A C s 1)$ :

i) DOCSs 1: The document space contains all axioms of the document space of Simple, DOCSs, but it does not contain any of the predicates. In addition, it contains a set of $s$ constants which name the knowledge topics T1, T2, Ts in the knowledge space. It also contains a finite set of predicates, stating the learning dependencies between these topics: depends $(\mathrm{Tj}, \mathrm{Tk})$, with $\mathrm{Tj} \_=\mathrm{Tk}$, means that topic $\mathrm{Tk}$ is required to understand $\mathrm{Tj}$. The documents are characterized by predicate keyword which assigns a nonempty set of topics to each of them, so $\forall D i \exists$ Tjkeyword $(D i, T j)$, but keep in mind that more than one keyword might be assigned to a same document.

ii) UMs1: The user model is the same as in Simple, plus an additional rule which defines that a topic $\mathrm{Ti}$ is assumed to be learned whenever the corresponding

document has been visited by the user. To this aim, Simple 1 uses the constant Learned. The rule for processing the observation that a topic has been learned by a

user is as follows (p obs is the abbreviation for "processing an observation"):

$$
\begin{gathered}
\forall U_{i} \forall T_{j}\left(\exists D_{k} \operatorname{keyword}\left(D_{k}, T_{j}\right) \wedge \text { obs }\left(D_{k}, U_{i} \text {, Visited }\right)\right. \\
\Rightarrow P_{-} \text {obs }\left(T_{j}, U_{i} \text { Learned }\right)
\end{gathered}
$$

iii) OBSs1: Are the same as in Simple. iv) ACs1: The adaptation component of Simple1 contains two further constants (w.r.t. Simple), representing new values for the learning state of a document [7] [4]. Such constants are: Might be understandable and will become understandable

Two more constants are added for representing new values for adaptive link annotation.

They are: Orange Icon and Yellow Icon. Such constants appear in the rules that describe the educational state of a document, reported hereafter. The first rule states that a document is recommended for learning if all the prerequisites to the keywords of this document have already been learnt:

$$
\begin{aligned}
& \forall U_{i} \forall D_{j}\left(\forall T_{k} \operatorname{keyword}(D j, T k) \Rightarrow\right. \\
& \left(\forall T_{l} \text { depends }\left(T_{k}, T_{l}\right)=\Rightarrow \text { p_obs }\left(T_{l}, U_{i} \text { L Learned }\right)\right. \\
& \Rightarrow \text { learning_state }\left(D_{j}, \quad U_{i}\right. \text { Recommended_ }
\end{aligned}
$$$$
\text { for_reading))) }
$$

The second rule states that a document might be understandable if at least some of the prerequisites have already been learnt by this user:

$$
\begin{array}{r}
\forall U_{i} \forall D_{j}\left(\forall T_{k} \operatorname{keyword}\left(D_{j}, T_{k}\right) \Rightarrow\right. \\
\left(\exists T_{l} \text { depends }\left(T_{k}, T_{l}\right) \Rightarrow\right. \\
\text { P_obs }\left(T_{b}, U_{i}, \text { Learned }\right)
\end{array}
$$

$\Lambda \neg$ learning state $\left(D_{j}, U_{b}\right.$ Recommended_for_reading)

$\Rightarrow$ learning state $\left(D_{j}, U_{b}\right.$, Might be understandable $\left.\left.)\right)\right)$

The third rule entails that a document will become understandable if the user has some prerequisite knowledge for at least one of the document's keywords:

$$
\begin{gathered}
\forall U_{i} \forall D_{j}\left(\exists T_{k} \text { keyword }\left(D_{j}, T_{k}\right) \Rightarrow\right. \\
\left(\exists T_{l} \text { depends }\left(T_{k}, T_{l}\right) \Rightarrow\right. \\
\operatorname{pobs}(T l, \text { Ui, Learned })
\end{gathered}
$$

$\Lambda \neg$ learning state $\left(D_{j}, U_{j}\right.$, Might be understandable)

$\Rightarrow$ Learning state $\left(D_{j}, U_{j}\right.$ Will become understandable $\left.)\right)$ )

Four rules describe the adaptive link annotation:

1) $U_{i} \forall D_{j}$ learning $\operatorname{state}\left(D_{j}, U_{i}\right.$ Recommended for reading)

$$
\Rightarrow \text { document annotation }\left(D_{j}, U_{i} \text {, Green Icon }\right)
$$

2) $\forall U_{i} \forall D_{j}$ learning state $\left(D_{j}, U_{i}\right.$ Will become । understandable)

$$
\Rightarrow \text { document annotation }\left(D_{j}, U_{i} \text { Orange Icon }\right)
$$

3) $\forall U_{i} \forall D_{j}$ learning state $\left(D_{j}, U_{i}\right.$, Might be understandable) 
$\Rightarrow$ document annotation $\left(D_{j}, U_{i}\right.$, Yellow Icon $)$

4) $\quad \forall \quad U_{i} \quad \forall \quad D_{j} \quad \neg \quad$ learning $\quad$ state $\left(D_{j}\right.$, $U_{i}$ Recommended_for_reading)

$$
\Rightarrow \text { document annotation }\left(D j, U_{i}\right. \text { Red Icon) }
$$

\section{CONCLUSION}

Present and the future research in e-learning system are on the intelligent learning systems. The platform for this is the Semantic Web and the Web Ontology's. One common assumption is that the Semantic Web can be made a reality by gradually augmenting the existing data (HTML/XHTML) by ontological annotations, derived from the on-machinereadable content This paper presents an intelligent e-learning system i.e., An Adaptive Educational Hyper media System which is based on the hypermedia system using hypertext link by traffic metaphor. This system is aimed at providing user required information effectively and efficiently. The aim of this study is to extend this model to other areas like ecommerce, Artificial intelligence problems for knowledge retrieval.

\section{REFERENCES}

[1] Zhiming Cui, Wei Fang, Xuefeng Xian, Shukui Zhang, and Pengpeng Zhao "Extension of OWL with Dynamic Fuzzy Logic"proc. International workshop on web based contents managementtechnologies [WCMT-2009] pp 67-76

[2] Fayed F. M. Ghaleb, 1Sameh S. Daoud, 2Ahmad M. Hasna, 2Jihad M. Jaam and Hosam F. El-Sofany "A Web-Based E-Learning System Using Semantic Web Framework" Journal of Computer Science 2 (8): 619626, 2006, ISSN 1549-3636, () 2006 Science Publications

[3] Nicola Capuano1, 3, Matteo Gaeta1, 3, Alessandro Micarelli1, 4 and Enver Sangineto2 "An Intelligent Web Teacher System for Learning Personalization and Semantic Web Compatibility".

[4] IWT: Intelligent Web Teacher. White Paper,CRMPA, 2002.

[5] Capuano N., De Santo M., Marsella M., Molinara M. and Salerno S. "Personalised Intelligent Training on the Web" Multimedia Systems and Applications Series, vol. 20, chap. 5, 2001.

[6] Capuano N., Gaeta M., Micarelli A. and Sangineto E. "An integrated Architecture for Automatic Course Generation". Proceedings of the IEEE International Conference on Advanced Learning Technologies, Kazan, Russia, 2002

[7] Felder R. M., "Learning and Teaching Styles in Engineering Education", Engr. Education 78 (7), 674-681, 1988.
[8] Matteo Baldoni1, Cristina Baroglio1, and Nicola Henze2" Personalization for the Semantic Web*” N. Eisinger and J. Maluszynski (Eds.): REWERSE 2005, LNCS 3564, pp. 173-212, 2005. c_SpringerVerlag Berlin Heidelberg 2005.

[9] http://swoogle.umbc.edu/

[10] Cardoso, J. and A. Sheth(2003). "Semantic e-Workflow Composion."Journal of Intelligent Information Systems (JIIS).21(3):191-225

[11] Kashyap, V. and A. Sheth (1996). "Semantic heterogeneity in global information systems: the role of metadata, context and ontologies". Cooperative Information Systems: Current Trends and Applications. M.Papzoglou and G.Schlageter. London,Uk, Academic Press: 139-178.

[12] Matteo Baldoni, C. Baroglio, and V. Patti. "Web-based adaptive tutoring: an approach based on logic agents and reasoning about actions". Artificial Intelligence Review, 22(1), September 2004.

[13] Fensel, D., F. van Harmelen, I. Horrocks, D.L. Mc Guinness and P.F. Patel-Schneider, 2001. OIL: An ontology infrastructure for the semantic web. IEEE Intelligent Systems, 16: 38-45.

[14] Matteo Baldoni, C. Baroglio, V. Patti, and L. Torasso. "Reasoning about learning object metadata for adapting scorm courseware". In L. Aroyo and C. Tasso, editors, Proc. of Int. Workshop on Engineering the Adaptive Web, EAW'04: Methods and Technologies for personalization and Adaptation in the Semantic Web, pages 4-13, Eindhoven, The Netherlands, August 2004.

[15] Rokou, F.P. et al., 2004. "Modeling web-based educational systems: process design teaching model". Educat. Technol. Soc., 7: 42-50.

[16] Adelsberger, H. et al., 2003. "The Essen model: A step towards a standard learning process". http://citeseer.ist.psu.edu/515384.html.

[17] T. R. Gruber. "A translation approach to portable ontologies". Knowledge Acquisition, 5(2):199-220, 1993.

[18] T. R. Gruber. "Toward principles for the design of ontologies used for knowledge sharing". Presented at the Padua workshop on Formal Ontology, March 1993, later published in International Journal of Human-Computer Studies, Vol. 43, Issues 4-5, November 1995, pp. 907-928.

[19] Charlie Abela, Matthew Montebello "A Personalized Service Discovery and Composition Framework" funded by the European Commission and by the Swiss Federal Office for Education and Science within the 6th Framework Programme project REWERSE number 506779

[20] R. Reiter. "A theory of diagnosis from first principles" Artificial Intelligence, 32, 1987.

[21] J.Lobo, G.Mendez, and S.R. Taylor. "Adding Knowledge to the Action Description Language" A. In proc. Of AAAI97/IAAF97, pages 454-459 Menlo park,1997.

[22] I. Horrocks, P. Patel-Schneider, H. Boley. S. Tabet, and B. Grosof.SWRL: "A semantic web rule language combining OWL and RuleML",200 